\title{
Antinuclear antibody studies in juvenile chronic arthritis
}

\author{
A M LEAK, B M ANSELL, AND S J BURMAN \\ Division of Rheumatology, Canadian Red Cross Memorial Hospital, Taplow and Clinical Research Centre, \\ Watford Road, Harrow, Middlesex
}

SUMmARY This study of children with chronic arthritis confirms that patients at greatest risk for chronic iridocyclitis have a young age of onset and positive antinuclear antibodies (ANA). Children who are ANA negative have a low risk of iridocyclitis. When it occurs it is at a significantly older age. No child developing juvenile chronic arthritis over 9 , even with positive ANA, had chronic iritis.

There was remarkable variation in ANA titres; these correlated with erythrocyte sedimentation rate, active arthritis, and iridocyclitis. Maximum ANA titres were often seen very early in the disease but at this time were usually not associated with a poor prognosis.

Histocompatibility antigens A2 and DRw8 were significantly increased, suggesting primary associations in pauciarticular arthritis with ANA. HLA-DR5 was associated with mild forms of arthritis and with absence of, or only mild, iritis. HLA-B15/w62 was associated with severe forms of eye involvement.

Chronic inflammatory arthritis in childhood is known to encompass several subgroups with differing clinical and laboratory findings. Splitting patients into groups may help in the assessment of long term prognosis and management. ${ }^{1}$

Antinuclear antibodies (ANA) have been detected by immunofluorescence in roughly $30 \%$ of children with juvenile arthritis. ${ }^{23}$ Patients who are ANA positive usually fall into one of three subgroups. The largest is children developing pauciarticular arthritis-that is, four or fewer joints affected-at a young age; they are predominantly girls and are prone to develop chronic iridocyclitis. ANA are also found among older children with a polyarthritis associated with IgM rheumatoid factor and less commonly among children with seronegative polyarthritis.

The occurrence of chronic iridocyclitis in seronegative juvenile chronic arthritis was first described by Ohm in $1910,{ }^{4}$ but it was not until 1974 that Schaller noted its close association with positive ANA. Possession of ANA, however, did not seem to predict whether a child's eye or joint disease would be mild or severe. ${ }^{3}$ The terms chronic iridocyclitis, iritis, and uveitis are used interchangeably.

We have examined the longitudinal relation between ANA titres and disease activity in joints and eyes and have also looked at histocompatibility antigens (HLA).

\section{Patients and methods}

One hundred children with seronegative juvenile chronic arthritis referred to Taplow between 1970 and 1980 were identified as having had either positive ANA or chronic iridocyclitis, or both. The course of their joint and eye disease was charted using the number of active joints and the number of cells seen in the anterior chamber of the eye as indicators of activity. Erythrocyte sedimentation rate (ESR) and all ANA results were noted.

These patients underwent a clinical review between November 1981 and October 1982, and blood was taken for HLA antigens as well as ESR and ANA.

Disease pattern. Although most children with ANA and iritis have a pauciarticular onset of arthritis, it can extend to involve more than four joints. To define those children with a spreading arthritis the following terms were used, the onset time being the first three months.

(1) Persistent pauciarticular: never more than four joints involved. 
(2) Pauciarticular in type: pauciarticular in onset and predominantly with four or fewer active joints, but up to a maximum of nine joints may be involved for periods of less than 12 months. This group includes children with more than four joints at onset who settle within a year to the pauciarticular pattern.

Children with pauciarticular pattern (both (1) and (2) above) were grouped as having mild arthritis.

(3) Extended pauciarticular: pauciarticular in onset but extending to involve more than four joints for periods of more than 12 months or to include more than nine joints in total.

(4) True polyarticular: more than four joints involved at onset and persistently thereafter.

Children with extended or polyarticular pattern ((3) and (4)) were classed as having severe arthritis.

Severity of chronic iridocyclitis. The overall severity was graded as follows:

(1) Mild: one to three bouts of iritis, settling rapidly with or without treatment.

(2) Moderate: chronic active iritis, usually requiring treatment but with periods of remission.

(3) Severe: secondary complications of cataract or glaucoma.

Laboratory data. ANA were measured by indirect immunofluorescence using rat liver substrate. Titres from our laboratory (in IU) and from other laboratories, where other results were used, were graded as shown in Table 1 using $1 / 40$ as a cut off point.

Clinical and laboratory data were tested non-

Table 1 Titres of antinuclear antibodies graded using 1/40 as a cut off point

\begin{tabular}{|c|c|c|c|c|}
\hline & \multicolumn{4}{|l|}{ Grade } \\
\hline & \multirow{2}{*}{$\frac{\text { Negative }}{0}$} & \multicolumn{3}{|l|}{ Positive } \\
\hline & & $I$ & 2 & 3 \\
\hline Titres & $1 / 10$ & $1 / 40-1 / 80$ & $1 / 160-1 / 640$ & $\geqslant 1 / 1280$ \\
\hline Taplow $1974 \rightarrow$ & 6 & 25 & $100-400$ & $\geqslant 1600$ \\
\hline Taplow $1970-73$ & 1 & 31 & 158 & $\geqslant 315$ \\
\hline
\end{tabular}

parametrically using Spearman's coefficient of rank correlation.

HLA typing was performed on all patients for HLA-A, B, C, and DR antigens using a panel of 240 established antisera and taking particular care with the cross reacting specificities DR5, DRw6, and DRw8. Controls for the HLA data were 192 random, normal unrelated subjects. For HLA statistical analysis six children were excluded, four on grounds of nationality and two because an affected sibling had been included.

Antigen frequencies between different groups of patients were compared using $2 \times 2$ contingency tables, and the significance of associations was assessed by means of $\chi^{2}$ calculations (with Yates's correction) and/or calculation of Fisher's exact probabilities. Few of the apparent antigen correlations with subgroups of patients remained significant when probabilities were corrected to allow for the number of comparisons performed, though their significance may be confirmed when viewed in the light of other studies. Probabilities quoted are therefore uncorrected.

\section{Results}

Duration of disease and sex. The mean duration of disease was 9.5 years with a range from 4.4 to 38 years. There were 75 girls and 25 boys, with no significant differences between the subgroups in the frequency of occurrence of ANA, nor severity of arthritis or iritis.

Disease pattern groups. There were 67 children with both ANA and iritis, 27 with ANA alone, and six with iritis alone (Table 2). Ninety patients had a pauciarticular onset of arthritis, and 25 remained persistently pauciarticular. Thirty four patients were classed as pauciarticular in type, and this included four patients with more than four joints involved at onset. Thirty five children had extended pauciarticular disease. Six were classed as true polyarticular, including one boy with systemic features.

Table 2 shows the distribution of ANA and iritis

Table 2 Distribution of antinuclear antibodies and iritis by disease pattern for 100 children with juvenile chronic arthritis

\begin{tabular}{|c|c|c|c|c|c|}
\hline & \multicolumn{2}{|l|}{ Mild } & \multicolumn{2}{|l|}{ Severe } & \multirow[b]{2}{*}{ Total } \\
\hline & $\begin{array}{l}\text { Persistent } \\
\text { pauciarticular }\end{array}$ & $\begin{array}{l}\text { Pauciarticular } \\
\text { in type }\end{array}$ & $\begin{array}{l}\text { Extended } \\
\text { pauciarticular }\end{array}$ & $\begin{array}{l}\text { True } \\
\text { polyarticular }\end{array}$ & \\
\hline \multirow{3}{*}{$\begin{array}{l}\text { Antinuclear antibodies and iritis } \\
\text { Antinuclear antibodies alone } \\
\text { Iritis alone }\end{array}$} & 14 & 25 & 23 & 5 & 67 \\
\hline & 10 & 9 & 8 & 0 & 27 \\
\hline & 1 & 0 & 4 & 1 & 6 \\
\hline
\end{tabular}


by disease pattern; there were no significant differences in the subgroups. Table 3 shows the even distribution of severity of eye involvement according to possession of ANA.

Age of onset. The mean age of onset of disease was 3.7 years with a range from 6 months to 15 years (Figure 1). Altogether, 79 had an onset under the age of 5 and 95 under the age of 10 . Only five (38\%) of the 13 children who developed arthritis aged 6 or more subsequently developed iritis compared with $22(81 \%)$ of the 27 with onset under 2 .

The mean age of onset was significantly less in the group with both ANA and iritis (3.0 years) than in those with ANA alone $(5 \cdot 1$ years $)(p=0 \cdot 002)$. All five patients with onset aged 10 or more were in the group positive for ANA but without eye disease. The children with iritis alone had an intermediate age of onset of $4 \cdot 1$ years.

The mean age of onset of uveitis was 5.8 years, but there was a significantly older age of onset in the ANA negative patients ( 14.0 years) compared with the ANA positive patients $(5 \cdot 1$ years $)(\mathrm{p}<0 \cdot 001)$.

The mean time between onset of arthritis and

Table 3 Distribution of severity of eye involvement according to possession of antinuclear antibodies in 100 children with juvenile chronic arthritis

\begin{tabular}{llrlrrr}
\hline $\begin{array}{l}\text { Antinuclear } \\
\text { antibodies }\end{array}$ & \multicolumn{2}{l}{ Severity } & of eye & involvement & Total \\
\cline { 2 - 6 } & None & Mild & Moderate & Severe & \\
\hline Positive & 27 & 18 & 16 & 33 & 94 \\
Negative & - & 2 & 0 & 4 & 6 \\
\cline { 2 - 6 } & 27 & 20 & 16 & 37 & 100 \\
\hline
\end{tabular}

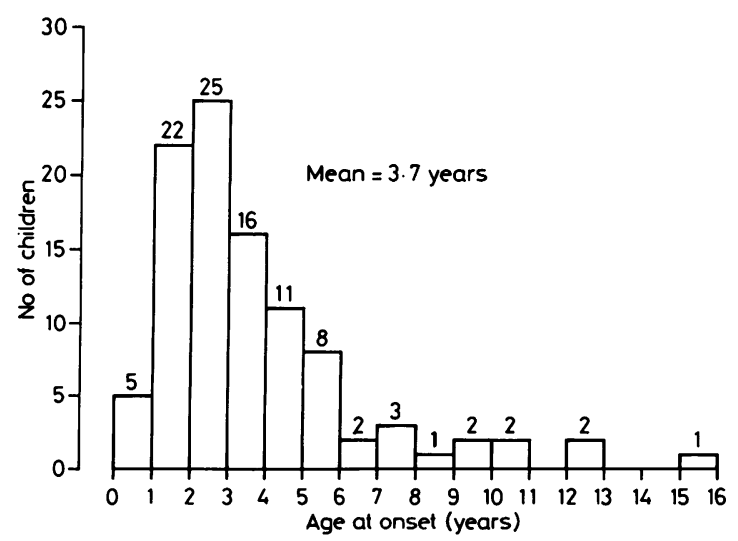

Fig. 1 Age of 100 children at onset of juvenile chronic arthritis.

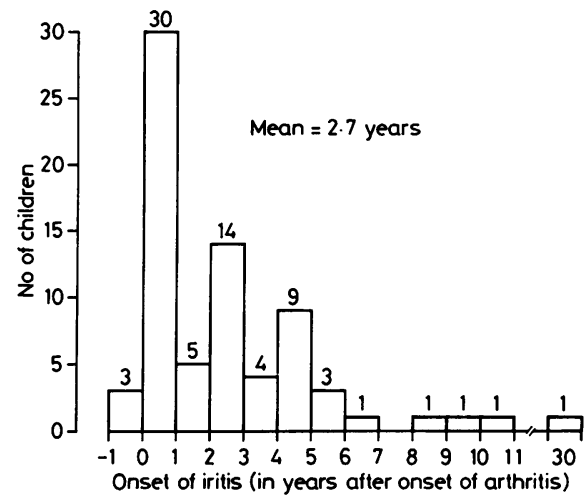

Fig. 2 Time between onset of arthritis and onset of iritis in 73 children.

discovery of iridocyclitis was 2.7 years (Figure 2). Three patients developed iritis before arthritis with a mean time difference of nine months.

When the severity of eye disease was assessed the age of onset of arthritis was not an indication of the severity of outcome, but there was a trend towards those with a late onset of iritis having relatively mild involvement, especially in the few patients where it developed more than five years after the arthritis.

Data evaluation. Data on the patients included 590 active joint and eye assessments and 415 ESR and 388 ANA estimations. There was a highly significant association of active joint count with high ESR $\left(p=0 \cdot 01 \times 10^{-4}\right)$. ANA titres also correlated with $\operatorname{ESR}(p=0.005)$, with active joints $\left(p=0.03 \times 10^{-5}\right)$, and with active iritis $(p=0.01)$.

At the two, five, and 10 year follow ups there were significant associations of both active joint count and ESR with overall severity of arthritis (all $p=0.002$ ), except for the ESR at two years. After 10 years of disease children with extended pauciarticular and true polyarticular arthritis still had 4.6 and $10 \cdot 3$ active joints, respectively.

Of the patients who had ANA titres estimated at these three follow ups, there were no significant $\mathcal{O}$ associations of mean ANA with severity of disease, N though the persistent pauciarticular group almost $N$ always had lower mean titres than other groups $\omega$ (range 0.5 at 10 years to 0.9 at 2 years). The extended pauciarticular group maintained their $\stackrel{O}{=}$ positive ANA (mean 1.2) up to 10 years and this $\stackrel{\overparen{D}}{\oplus}$ paralleled their persistent mean active joint count of 4.9 and ESR of 37.5 .

At five years ANA titres correlated with active joint counts $(p=0.03)$, activity of iritis $(p=0.025)$, and $\operatorname{ESR}(p=0 \cdot 006)$. These associations did not 
reach significance at either two or 10 years when there were fewer results available for analysis.

Variation in ANA titres. There was considerable variability in the readings recorded in the individual patients. Six children were persistently ANA negative and 43 persistently ANA positive, although only seven had the same titre at each recording. Fifty one had at least one negative ANA during the course of their disease, including 17 who were negative when first tested. Thirty two children who had become ANA negative at the last follow up showed a trend towards milder eye and joint involvement, which had often become inactive.

First ANA recorded. The first ANA titre in each patient who then or subsequently became ANA positive was graded as described in Table 1 . The mean titre was highest $(2.10)$ in children tested within three months of onset falling to 1.87 in the first year of disease and 1.43 overall.

Maximum ANA recorded. The maximum ANA recorded in each patient was charted and most $(54 \%)$ had a grade 2 positive ANA, with $18 \%$ having grade 1 only and $22 \%$ having a maximum grade 3 . The proportion of patients with strongly positive ANAs (grade 3 ) was significantly greater in the two groups with severe arthritis than in those with milder disease, while the proportion did not vary significantly between groups according to severity of uveitis.

Among the 22 patients with grade 3 positive ANAs the mean time from date of onset of disease to maximum ANA was only 1.5 years in those with mild arthritis compared with 5.0 years in the severely affected group $(p=0 \cdot 04)$.

ANA before or at onset of chronic iridocyclitis. Of the 67 patients with ANA and iritis, only 13 had had ANA tested more than three months before its onset. Eight of 11 who had been previously positive remained so and two who had been initially ANA negative became positive at the time of onset. Of a further 25 patients who had ANA tested at or within three months of onset of iritis, 20 were positive, and three of the other five became positive when the test was repeated within six months.

Chronic iridocyclitis with negative ANA. Of these six patients (Table 3), three were girls and three boys. All had had at least three negative readings, including ANA at the time of onset of iritis in four and during periods of activity in four. Three patients had some atypical features of disease with one having bouts of acute as well as chronic iridocyclitis.
Table 4 Prevalence of HLA antigens in 94 children with juvenile chronic arthritis and 192 control patients

\begin{tabular}{llcl}
\hline Antigen & Patient $\%$ & Control $\%$ & $p$ Value* \\
\hline A2 & $76 \cdot 6$ & $46 \cdot 8$ & $0 \cdot 1 \times 10^{-5}$ \\
B15/w62 & $21 \cdot 3$ & $9 \cdot 3$ & $0 \cdot 004$ \\
DR5 & 30.9 & $14 \cdot 1$ & $0.09 \times 10^{-2}$ \\
DRw6 & $38 \cdot 3$ & $22 \cdot 4$ & $0 \cdot 004$ \\
DRw8 & 39.4 & $2 \cdot 7$ & $0 \cdot 1 \times 10^{-12}$ \\
\hline
\end{tabular}

*Fisher's exact test.

HLA antigens. There were significant increases in HLA antigens A2, B15/w62, DR5, DRw6, and DRw8 in our children compared with controls (Table 4). A2 and DRw8 were found throughout the group with no variation according to severity of arthritis. The increases in B15/w62, DR5, and DRw6 were only in the two groups with mild arthritis with no significant increase in the severely affected children when compared with controls.

The frequency of the A2 antigen was roughly the same in all grades of severity of uveitis. The frequency of DR5 was significantly higher in that group of patients with no or only mild eye disease than among controls $\left(p=0.03 \times 10^{-3}\right)$.

The frequency of DRw8 was increased above controls in all categories of eye involvement. The frequency, however, was significantly higher among patients with mild or no iritis than among patients with moderate or severe eye disease $(p=0 \cdot 01)$.

In children with iritis there was an increase in B15/w62 compared with those without iritis. In those with moderate and severe iritis this was significant even after correction for the number of $B$ antigens tested $\left(p=6.9 \times 10^{-4}\right)$.

\section{Discussion}

In young children with pauciarticular onset juvenile arthritis the association of ANA with chronic iritis and the HLA anitgens DR5 and DRw8 has been well documented. ${ }^{2}{ }^{35-10}$ In previous studies ANA have been shown in between $4 \%^{11}$ and $88 \%^{3}$ of patients. ANA has only been measured, however, at one point, sometimes very early in the disease ${ }^{2}$ and sometimes late, when most children had inactive arthritis. ${ }^{11}$ There were varying groups of children in each paper, including seropositive arthritis in five, ${ }^{2} 712-14$ and only two groups particularly studied those with iritis. ${ }^{3}{ }^{15}$ This study has selected patients with a very high incidence of positive ANA $(94 \%)$.

We confirm the very young age of onset of $3 \cdot 0$ years in those with both ANA and uveitis; this was significantly younger than those children with positive ANA who did not develop eye disease. The 
mean time between onset of arthritis and discovery of iritis was 2.7 years, but $45 \%$ of children developed it within 12 months of the onset of their arthritis. We suggest, therefore, that during this early period patients at risk should have slit lamp examinations at least every three months to detect iridocyclitis.

Previous studies have usually measured ANA at only one point. Hanson noted considerable variability in ANA positivity and titres over time but did not mention any associations. ${ }^{16}$

In those studies that looked for a correlation between disease activity and ANA titres, three found no relation with active arthritis, ${ }^{367}$ and only one correlated complement fixing granulocyte specific ANA in undiluted serum with both disease activity and high ESR. ${ }^{5}$ Similarly, three reports showed no relation between ANA titres and activity of iritis. ${ }^{35} 15$ One group found that eye involvement, rather than current activity, correlated with complement fixing ANAs in the small number of patients studied. ${ }^{15}$

In this longitudinal study we have been able to show a greater variation in ANA titres and would suggest that by taking serial estimations of ANA at different stages in the course of the disease a more sensitive relation with active joints, ESR, and active iritis has emerged. A high ANA titre in the early stages of disease was not uncommon and tended to be associated with milder forms of arthritis, particularly if later ANA titres had fallen to grade 1 positive or negative. Persistent moderately positive ANAs or those rising to maximum titres after several years of disease were often associated with more severe arthritis.

A positive ANA in any child with seronegative chronic arthritis is a marker for the possible development of uveitis, whether the ANA is present initially or develops several months or years into the disease. As many children carry ANA in the absence of uveitis, this is obviously not a specific stimulus to ANA formation, and we do not know the nature of the nuclear antigens involved. There certainly seems to be a difference between the ANA in juvenile chronic arthritis and that in patients with systemic lupus erythematosus, ${ }^{14}$ and further immunological studies may help to characterise the antigens involved.

Our HLA studies found a very high frequency of HLA-A2, suggesting a primary association with all types of pauciarticular onset arthritis, whether associated with ANA or iritis, or both. The positive associations with the DR antigens 5, w6, and w8 are also striking, and the increase in DRw8 to a frequency 15 times higher than in the controls suggests the presence of a linked disease gene conferring susceptibility to pauciarticular onset ${ }^{\circ}$ arthritis associated with ANA. By contrast, DR5 $\overrightarrow{\vec{F}}$ and DRw6 are only increased in the mild forms and not in the more severe arthritis.

The antigens DR5 and DRw8, present in the $\frac{\overline{\frac{\sigma}{\omega}}}{\overline{0}}$ highest frequencies, showed a significant trend to $\overrightarrow{\mathbb{D}}$ associations with the absence of, or only mild, iritis despite being linked to positive ANA. The finding $\mathscr{C}$ of B15/w62 being associated also with mild arthritis $\vec{O}$ but with the more severe forms of eye disease is interesting and will require confirmation from other $\vec{\sigma}$ groups.

\section{References}

1 Schaller JG. Juvenile rheumatoid arthritis. Pediatr Ann

1982;11:375-82.
Petty RE, Cassidy JT, Sullivan DB. Clinical correlates of $\vec{\infty}$ antinuclear antibodies in juvenile rheumatoid arthritis. J Pediatr 1973;83:386-9.

3 Schaller JG, Johnson GD, Holborow EJ, et al. The association of antinuclear antibodies with the chronic iridocyclitis of $\pi$ juvenile rheumatoid arthritis (Still's disease). Arthritis Rheum $\frac{D}{D}$ 1974;17:409-16.

4 Ohm J. Bandformige hornhaut trubung bei einenneuen jahrigen madchen. Klin Monatsbl Augenheilkd 1910;48:102-43.

5 Hoyeraal HM. Granulocyte reactive antinuclear factors in $\overrightarrow{c 0}$ juvenile rheumatoid arthritis. Scand J Rheumatol 1976;5:84-90. œ

6 Rossen RD, Brewer EJ, Person DA, et al. Circulating immune? complexes and antinuclear antibodies in juvenile rheumatoid $\square$ arthritis. Arthritis Rheum 1977;20:1485-90.

7 Permin H, Horbov S, Wiik A, Knudsen JV. Antinuclear antibodies in juvenile chronic arthritis. Acta Paediatr Scand 1978;67:181-5.

* Glass D, Litvin D, Wallace K, et al. Early-onset pauciarticular $\frac{\mathrm{O}}{\mathbb{Q}}$ juvenile rheumatoid arthritis associated with human leukocyte antigen-DRW5, iritis and antinuclear antibody. J Clin Invest $\overrightarrow{\overline{0}}$ 1980;66:426-9.

9 Forre O, Dobloug JH, Hoyeraal HM, Thornsby E. HLA $\frac{3}{\supset}$ antigens in juvenile arthritis: genetic basis for the different subtypes. Arthritis Rheum 1983;26:35-8.

10 Miller ML, Fraser PA, Jackson JM, et al. Inherited predisposi- ֻิ tion to iridocyclitis with juvenile rheumatoid arthritis: selectivity $\frac{\Omega}{\Omega}$ among HLA-DR5 haplotypes. Proc Natl Acad Sci USA 1984;81:3539-42.

11 Bluestone R, Goldberg LS, Katz RM, et al. Juvenile rheumatoid arthritis: a serologic survey of 200 consecutive patients. J Pediatr 1970;77:98-102.

12 Kornreich HK, Drexler E. Hanson V. Antinuclear factors in $\mathrm{O}$ childhood rheumatic diseases. J Pediatr 1966;69:1039-45.

13 Rudnicki RD, Ruderman M, Scull E, et al. Clinical features and $\frac{D}{O}$ serological abnormalities in juvenile rheumatoid arthritis. Arthritis Rheum 1974;17:1007-15.

14 Alspaugh MA, Miller JJ. A study of specificities of antinuclear $\widehat{N}$ antibodies in juvenile rheumatoid arthritis. $J$ Pediatr $N$ 1977;90:391-5.

15 Egeskjold EM, Johansen A, Permin H, et al. The significance of antinuclear antibodies in juvenile rheumatoid arthritis associ- $\sigma$ ated with chronic bilateral iridocyclitis. Acta Paediatr Scand 1982;71:615-20.

Correspondence to $\operatorname{Dr}$ A M Leak, Division of Rheumatology, $\stackrel{\mathbb{D}}{+}$ Clinical Research Centre, Northwick Park Hospital, Harrow, $\square$ Middlesex HA1 $3 \mathrm{UJ}$.

Received 11 September 1985 